\title{
Biomarkers of ocular surface disease using impression cytology
}

\author{
Suzanne Hagan*,1 \\ ${ }^{1}$ Vision Sciences, Dept. of Life Sciences, Glasgow Caledonian University, G4 OBA, Scotland, UK \\ * Author for correspondence: Tel.: +44 141331 8657; Fax: +44 141331 3387; suzanne.hagan@gcu.ac.uk
}

Impression cytology (IC) is a technique which permits the retrieval of the outermost layer of ocular surface cells via the use of various types of filters. It is a minimally invasive method of evaluating human conjunctival epithelial cell morphology in the diagnosis of dry eye disease, a common and distressing disorder associated with ageing, contact lens wear, autoimmune disorders and refractive (LASIK) surgery. IC may also be utilized in the diagnosis of other ocular diseases, such as keratoconus and thyroid orbitopathy. More recently, IC has been utilized for the subsequent investigation of gene and protein expression of conjunctival cells in order to identify novel diagnostic biomarkers and to further our understanding of the mechanisms underlying ocular surface disease. This review will therefore examine the literature concerning the role of IC in identifying cellular markers of eye disease, systemic diseases with ocular involvement and potential novel therapeutic targets.

First draft submitted: 11 April 2017; Accepted for publication: 4 September 2017; Published online: 28 November 2017

Keywords: biomarkers $\bullet$ conjunctiva $\bullet$ dry eye disease $\bullet$ impression cytology $\bullet$ inflammation $\bullet$ ocular surface $\bullet$ personalized medicine

Impression cytology (IC) is a minimally invasive method of evaluating human ocular surface cells and is considered as the 'gold standard' technique for assessing cell morphology. Introduced by Thatcher et al. [1] and Egbert et al. [2], IC is an easy-to-use technique of directly retrieving (mainly conjunctival) cells, using various filter types which are gently applied to exposed (interpalpebral) conjunctival surfaces. The filters used include cellulose acetate [3], polycarbonate (Biopore, Millipore UK Ltd, Watford, UK) [4], nitrocellulose [5] or polyethersulfone (PES) [6] which are gently applied to exposed (interpalpebral) conjunctival surfaces. Recently, a more standardized tool for IC sampling has been introduced (Figures $1 \& 2$ ). The Eyeprim is a new single-use PES filter sampling medical device for retrieval of conjunctival cells (Opia Technologies, France) and a recent study by Lopez-Miguel et al. [7] performed an analysis of this tool versus the traditional IC method (PES). On a cohort of 20 healthy individuals, they extracted and compared RNA from both devices and found no significant differences in the mean yield ( $\mathrm{p}$ $\geq 0.45)$, the total RNA amount $(\mu \mathrm{g})$, or the amount normalized to membrane area [7]. Although an earlier study of the Eyeprim [8], suggested that it recovered more cells than traditional IC, Lopez-Miguel et al. [7] did not observe differences in the RNA collected between this device and PES.

Historically, IC has been used to analyze pathological changes to conjunctival cells for indicators of squamous metaplasia in ocular surface disease (OSD) [9]. More recently, however, researchers have used IC to examine specific markers of inflammation, for example, HLA-DR, ICAM-1 and CD8 [10-12], and to examine novel biomarkers of dry eye disease (DED). The latter is performed in an attempt to understand the mechanisms underpinning DED pathophysiology and is now feasible, due to vast improvements in the assay sensitivities for gene and protein examination [13]. As a result, IC membranes can be subsequently analyzed using techniques such as immunofluorescent staining, flow cytometry, multiplex bead immunoassays and real-time PCR (qPCR) [14-17]. For example, recent research by our group on RNA extracted from Eyeprims of DED subjects demonstrated adequate RNA levels to perform qPCR for a number of inflammatory markers, including $p 38 M A P K-\alpha, I L-1 \beta, I L-8, M C P-1$ and $M M P-9$ [18]. This study showed that, in subjects with DED, all five proinflammatory genes were significantly upregulated, compared with normal subjects $(\mathrm{p}<0.001)$. 

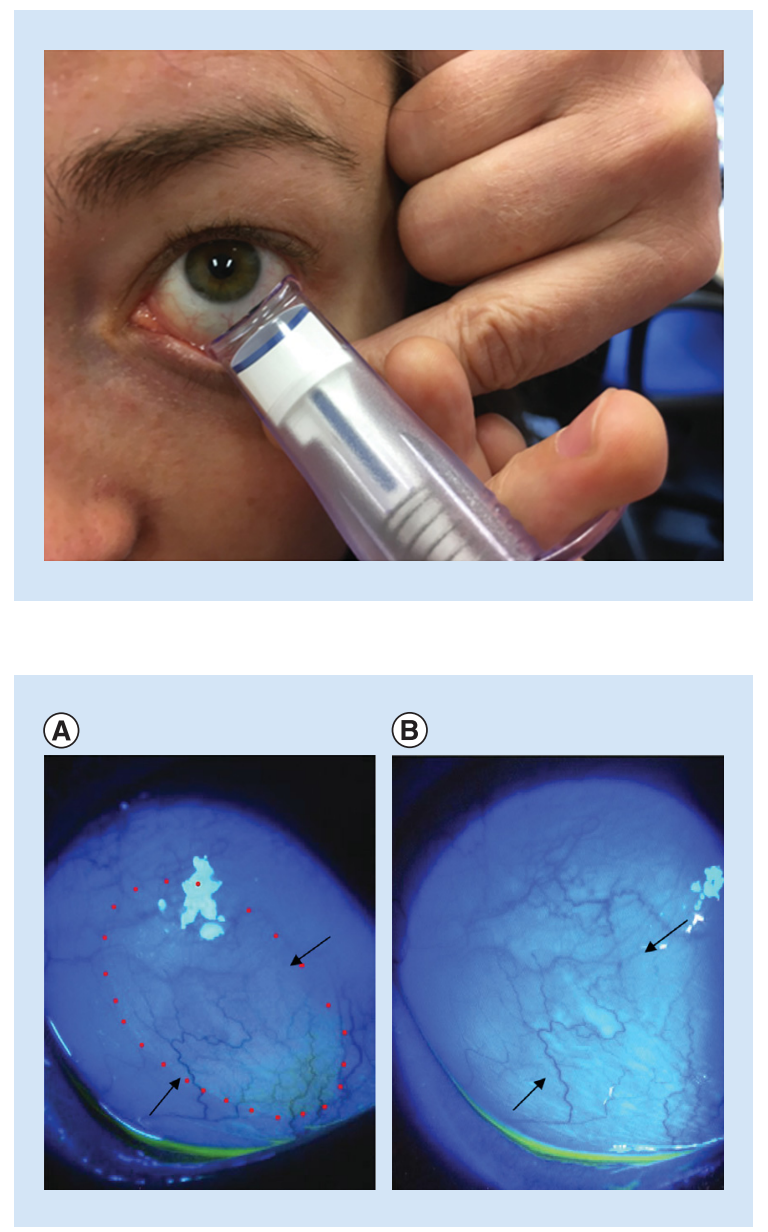

Figure 1. Conjunctival impression cytology sampling using the Eyeprim device.
Figure 2. Fluorescein staining of the conjunctiva. (A) Immediately after Eyeprim sampling (red dots show outer limits of staining) and (B) at $24 \mathrm{~h}$ post-sampling (no fluorescein staining visible). Note the re-epithelialisation of the ocular surface within 1 day of cell retrieval.

It is therefore anticipated that biomarkers identified by IC will serve as future novel diagnostic tools (for better stratification of patients), and in measuring outcomes of targeted clinical therapies. Together, it is hoped that the use of IC-validated biomarkers may help to develop a more personalized approach to diagnosing and treating ocular, and possibly some systemic diseases.

\section{Impression cytology \& ocular surface disease}

As mentioned, IC has traditionally been used to identify morphological changes (e.g., squamous metaplasia), in OSD, primarily in DED. However, subsequent IC studies have assessed other OSDs and have noted substantial conjunctival goblet cell loss and changes to epithelial cell size. For example, in 1986 Wittpenn et al. [19] first demonstrated reduced goblet cell numbers and increased epithelial cell size of the conjunctiva in vitamin A deficiency-induced xerophthalmia in children, and suggested a role for IC in early detection of this condition. Since then, various groups have used IC to assess the effects of DED therapies on the ocular surface, including the effects of preservatives in ophthalmic medications and autologous serum [20-22]. A summary of potential OSD-related biomarkers using IC can be found in Table 1.

\section{Dry eye disease \& squamous metaplasia}

In DED, squamous metaplasia is defined as the transition from a nonkeratinized, stratified epithelium (e.g., conjunctiva and cornea) to a nonsecretory, keratinized epithelium. In the conjunctival epithelium, squamous metaplasia presents as a continuum of changes, including reduction/loss of goblet cells (hence mucin deficiency) and gradual alterations of nongoblet epithelial cells (i.e., increased keratinization and stratification), as well as cellular enlargement and an increased cytoplasmic/nuclear ratio [23]. Squamous metaplasia can thus be considered as altered differentiation and this process is thought to be initiated by diminished aqueous tear production, resulting in tear fluid hyperosmolarity. 


\begin{tabular}{|c|c|c|c|}
\hline Biomarker & Disease & Authors & Ref. \\
\hline $\begin{array}{l}\text { SPRR1B, } \\
\text { IL-1 } \beta, \text { PAX6 } \\
\text { HLA-DR }\end{array}$ & SS-DE & $\begin{array}{l}\text { McNamara et al. (2014) } \\
\text { Jirsova et al. (2014) }\end{array}$ & $\begin{array}{l}{[47]} \\
{[22]}\end{array}$ \\
\hline EGFR, IL-6, IL-9, NAMPT, CD8 & oGvHD & $\begin{array}{l}\text { Cocho et al. (2015) } \\
\text { Eberwein et al. (2013) }\end{array}$ & $\begin{array}{l}{[43]} \\
{[12]}\end{array}$ \\
\hline IL-1 $\beta$, IL-8, MMP-9 TGF- $\beta 1$ & Blepharitis and MGD & Zhang et al. (2015) & [39] \\
\hline $\begin{array}{l}\text { HLA-DR, ICAM-1, } \\
\text { MUC1, MUC2, MUC4, MUC5AC, } \\
\text { IL-6 }\end{array}$ & Generic DED/ OSD & $\begin{array}{l}\text { Aronni et al. (2006) } \\
\text { Corrales et al. (2011) } \\
\text { López-García et al. (2016) } \\
\text { Zhang et al. (2013) }\end{array}$ & $\begin{array}{l}{[32]} \\
{[33]} \\
{[14]} \\
{[34]}\end{array}$ \\
\hline $\begin{array}{l}\text { Eotaxin-1, }-2, \&-3, \text { Hemopexin } \\
\text { CCL-20 }\end{array}$ & Ocular allergies: VKC, AKC & $\begin{array}{l}\text { Shoji et al. (2009) } \\
\text { Pong et al. (2011) } \\
\text { Inada et al. (2014) } \\
\text { Shiraki et al. (2016) }\end{array}$ & $\begin{array}{l}{[51]} \\
{[52]} \\
{[53]} \\
{[54]}\end{array}$ \\
\hline $\begin{array}{l}\text { HLA-DR, ICAM-1 } \\
\text { IL-1 } \alpha, \text { IL-1 } \beta, \text { IL-6. }\end{array}$ & Thyroid orbitopathy & $\begin{array}{l}\text { Pawlowski et al. (2014) } \\
\text { Hsu et al. (2016) }\end{array}$ & $\begin{array}{l}{[62]} \\
{[15]}\end{array}$ \\
\hline
\end{tabular}

\section{Dry eye disease \& inflammatory markers}

$H L A-D R$

As well as assessing morphological changes in squamous metaplasia, IC can be used to quantify inflammatory markers, such as the MHC Class II antigen HLA-DR. HLA-DR was found to be induced by exposure of conjunctival cells to hyperosmolarity [24]. HLA-DR is thus considered a proinflammatory marker, and it is specifically upregulated in patients with severe DED. In 1992, Baudouin et al. showed that IC could specifically detect HLA-DR expression in human conjunctival cells [11]. Then, in 1997 Baudouin demonstrated HLA-DR quantification via IC, using flow cytometry [25]. More recently, Yafawi et al. [26] further demonstrated the usefulness of HLA-DR (via IC) as a biomarker of OSD, in a validation study to assess its reliability by IC and flow cytometry. To assess specificity, 12 donors were divided into groups; normal, mild DED, moderate DED or severe DED (three per group). The authors reported higher HLA-DR expression in moderate DED (19.8\% over normals) and severe DED $(23.3 \%$ over normals), while mild DED showed a $10.8 \%$ increase over normal samples. The authors concluded that HLADR could serve as an inflammatory biomarker which correlated with DED severity, and that IC was a simple and reproducible technique [26].

It should be noted that quantitative HLA-DR detection by IC has been used in several DED clinical trials. Indeed, Epstein et al. [27] used a multicenter study to establish a standard operating procedure for utilizing IC and HLA-DR as an objective DED inflammatory biomarker, using duplicate samples from dry eye and normal subjects. The study found that expression of HLA-DR expression may be a reliable and objective inflammatory biomarker for use in multicenter clinical trial studies. More recently, Brignole-Baudouin [16] undertook an investigation of HLA-DR alongside clinical signs and symptoms, using data from 311 patients with moderate-to-severe DED from three clinical studies. In DED, clinical signs and symptoms rarely correlate, making diagnosis more difficult for the clinician. The authors reported a significant correlation between the clinical test of corneal fluorescein staining and HLA-DR ( $r=0.26$ and 0.30 , respectively), as well as with a tear production test (Schirmer's test; $r=-0.20$ ), albeit to a lesser degree. This group concluded that, although further work is necessary, HLA-DR expression may serve as a useful measure of eye surface inflammation and DED.

Furthermore, in terms of using IC and HLA-DR to assess DED therapies, recent research analyzed 20 aqueous deficient dry eye subjects who were exposed to low humidity, then compared the efficacy of artificial tears versus $0.1 \%$ dexamethasone [28]. IC membranes were analyzed for changes in $H L A-D R$ RNA expression by digital PCR and this group noted less irritation in those treated with dexamethasone versus artificial tear use, as well as reduced $H L A-D R$ expression (both $\mathrm{p}=0.01$ ) [28]. Topical cyclosporine (CsA) significantly reduced HLA-DR expression in patients with DED [29], and other work has demonstrated that oral supplementation of omega- 3 and omega- 6 fatty acids decreased HLA-DR expression in conjunctival cells of DED patients [30]. These studies indicate how IC 
may be used, not only in determining levels of ocular surface inflammation, but also in monitoring the outcome of various therapies.

\section{ICAM-1}

Similar to HLA-DR, ICAM-1 (CD54) is upregulated on the conjunctival epithelium in ocular surface inflammation and could represent a potential biomarker detectable by IC [31]. Although far fewer studies have been performed on this molecule than HLA-DR with regards to IC and DED, research in 2006 assessed ICAM-1 in subjects with severe dry eye [32]. They reported increased ICAM-1 expression (by immunofluorescent staining) in dry eye subjects versus controls. Moreover in dry eye, ICAM-1 staining had an inverse relation to number of goblet cells ( $\mathrm{p}<0.01$; rho $=-0.852$ ) and tear production (Schirmer's test; $\mathrm{p}<0.01$; rho $=-0.926$ ). Once again, a combined approach of IC and inflammatory biomarkers can be used to good effect in assessing dry eye.

\section{Dry eye disease \& mucins}

Mucins are the glycoproteins secreted by conjunctival goblet cells, which form the innermost layer of the tear film, the mucous layer. With the increasing improvements in sensitivities of assays, qPCR has been utilized to ascertain mucin changes at the gene level in IC membranes. For example, Corrales et al. [33] undertook a prospective study of patients with moderate-to-severe dry eye compared with healthy subjects. Following installation of topical anesthetic, two halves of a PES filter were applied to the superior and superotemporal bulbar conjunctiva of the most affected eye. RNA extraction and qPCR were performed for MUC1, MUC2, MUC4, and MUC5AC and MUC7, and the former four genes were found to be significantly lower in the conjunctiva of dry eye patients than controls (all $\mathrm{p}<0.0001$ ). The authors surmised that, of the genes assessed, MUC1 demonstrated the highest specificity and sensitivity (87.5 and 83.3\%, respectively), suggesting its potential as a diagnostic marker of DED [33]. More recent work on mucin expression in DED [14] also used this technique (qPCR) to assess the effects of autologous serum eye drops on conjunctival expression of MUC5AC. In a prospective, interventional study of various OSDs [14], 38 eyes $(\mathrm{n}=19)$ were assessed pre- and postautologous serum treatment ( 6 weeks). The authors reported increased MUC5AC expression which was related to conjunctival involvement (pretherapy), as well as diminished squamous metaplasia and increased goblet cells post-therapy $(\mathrm{p}<0.01)$. The researchers concluded that qPCR may be a new, reliable and objective means to assess the efficacies of DED therapeutics. They did, however, add a caveat that much larger patient cohorts were necessary, alongside longitudinal studies, in order to further understand the long-term effects of autologous serum.

In terms of protein analysis, Zhang et al. [34] utilized immunolabeling of IC membranes from 66 DED subjects, once again to assess MUC5AC, as well as the proinflammatory cytokine IL-6. This group reported lower MUC5AC in the severe symptom group, versus mild and moderate symptom groups (both $p<0.001$ ), while IL- 6 was increased in the severe symptom group (vs the mild and moderate symptom groups; both $\mathrm{p}<0.001$ ). Furthermore, a negative correlation ( $\mathrm{p}<0.001)$ was noted between MUC5AC and the scores for the OSD index (OSDI, a measure of patient-reported DED symptoms). Conversely, IL-6 was positively correlated with OSDI ( $\mathrm{p}<0.001)$. The authors concluded that there was an association between reduced mucin levels and increased IL-6 levels in DED, which correlated to severity of symptoms. These protein data appear to agree with both of the aforementioned qPCR studies $[14,33]$ in demonstrating reduced levels of MUC5AC in subjects with DED.

\section{Blepharitis \& meibomian gland dysfunction}

Blepharitis is the general term for chronic inflammation of the eyelids [35]. The etiology of blepharitis is not completely understood, however, as it is multifactorial in nature and usually occurs as a complication of other conditions, such as bacterial infections, atopy, diabetes, seborrhea and some parasites, such as demodex, as well as being associated with meibomian gland dysfunction (MGD) [36]. Extensive literature searches did not yield similar numbers of recent IC articles specifically on blepharitis, as for other DED subtypes. Although, conjunctival IC work by Karalezli et al. [37] on patients with seborrheic dermatitis revealed significantly fewer conjunctival goblet cells in this group compared with healthy controls $(\mathrm{p}<0.001)$. Furthermore, another study of 18 dry eye subjects (including some with presentations of blepharitis), underwent treatment with a therapeutic ocular surface medium (a tear replacement therapy) for up to 2 months [38]. In the randomized control trial, therapeutic ocular surface medium was found to significantly improve the blepharitis score $(p=0.002)$, as well as the conjunctival IC score $(p=0.028)$. While this was not conclusive evidence of a solely blepharitic/MGD group (mixed DED subtypes were used), it does indicate studies have been performed which include this specific dry eye demographic [38]. 
Another study [39] used IC and qPCR of the tarsal conjunctiva (and eyelid margin cells) to measure changes in the inflammatory mediators $I L-1 \beta, I L-8, M M P-9$ and TGF- $\beta 1$ of MGD patients $(\mathrm{n}=16)$ versus eight controls (preand post-azithromycin therapy). In this study of patients with MGD-related posterior blepharitis and conjunctival inflammation $(\mathrm{n}=16)$ treated with azithromycin, this group found that pretreatment MGD patients revealed significantly increased mRNA expression of $I L-1 \beta, I L-8$ and $M M P-9$ (all $\mathrm{p}<0.001$ ), and significantly reduced levels of TGF- $\beta 1$ ( $\mathrm{p}<0.02)$, when compared with healthy subjects. Following 2 weeks of azithromycin treatment, the mean mRNA expression of $I L-1 \beta, I L-8$ and $M M P-9$ was significantly diminished ( $\mathrm{p}<0.001 ; \mathrm{p}=0.002$ and $\mathrm{p}<0.001$, respectively), while $T G F-\beta 1$ was significantly increased $(\mathrm{p}=0.002)$. After 4 weeks of azithromycin treatment, expression of $I L-1 \beta, I L-8$ and $M M P-9$ were further reduced or remained lower $(I L-1 \beta ; \mathrm{p}<0.001 ; I L-8$, $\mathrm{p}<0.001 ; M M P-9 ; \mathrm{p}<0.001)$, and TGF- $\beta 1$ mRNA levels remained higher $(\mathrm{p}=0.001)$. This study concluded that IC is a simple and non-invasive technique to sample ocular surface cells, which permitted assessment of pro-inflammatory mediator expression pre- and post-topical azithromycin therapy [39].

\section{Ocular graft-versus-host disease}

Graft-versus-host disease (GvHD) is a condition that may occur following a hematopoietic (stem cell i.e. bone marrow) transplant, whereby white blood cells from the donated tissue treat the recipient's body as foreign, and thus immunologically attack the host's cells. Numerous organs are affected, for example, the liver, gut, skin and lungs, among others. Although there is an ocular-specific form, termed ocular GvHD (oGvHD), which may precede other vital organ damage. In oGvHD, the ocular surface tissues are predominantly affected, presenting as severe dry eye as a direct consequence of immune-mediated lacrimal gland (and conjunctival) destruction [40]. Recently, IC has also been used to assess morphological changes and novel biomarkers in subjects with oGvHD. For example, $\mathrm{Na}$ et al. [41] investigated subjects with chronic GvHD following allogeneic hematological stem cell transplantation that had been diagnosed with refractory DED. Alongside a number of other tests (including OSDI, Schirmer, tear break-up time [TBUT], tear osmolarity and cornea staining score), IC was performed to measure grade and goblet cell density pre- and postautologous serum therapy ( 4 weeks). This group reported that, as well as improvement in the other tests, improved IC grade and goblet cells density improved from 3.4 to 2.4, and from 90.6 to $122.6 \mathrm{cell} / \mathrm{mm}^{2}$, respectively [41]. A similar study, albeit without autologous serum therapy, also used IC on $40 \mathrm{GvHD}$ subjects with and without ocular involvement and they noted DED in 30 of the subjects and abnormal cell morphologies in $75 \%$ of all GvHD IC samples [42]. Of those with abnormal IC, a significant number were from subjects with oGvHD (91.7\%) versus those without oGvHD $(67.9 \% ; \mathrm{p}=0.024)$. This work indicated the utility of IC, alongside other measures (OSDI score, corneal involvement and Schirmer-I test) to ascertain levels of ocular morbidity in GvHD. Although the authors noted that cellular abnormalities were also observed in eyes without oGvHD.

Research by Cocho et al. [43] performed an analysis of 84 genes from IC samples of $20 \mathrm{oGvHD}$ patients and 14 healthy individuals. Following exposure to standardized environmental conditions, samples were retrieved and genes assessed via qPCR. Of these genes, 34 showed significant differences in expression. That is, in GvHD samples, increased expression of IL-6, IL-9, CCL24, CCL18, IL-10, IFN- $\gamma$ and CCL2 was noted (>sixfold); moderate overexpression of 26 genes was demonstrated (two to sixfold), while EGFR was downregulated (2.63fold). Following generation of a receiver operator curve, from a panel specifically made up of EGFR, IL-6, IL-9 and $N A M P T$, the researchers concluded that these four genes showed the greatest potential as oGvHD diagnostic biomarkers [43].

In terms of protein analysis, Eberwein et al. [12] has performed immunostaining of conjunctival IC membranes for HLA-DR, cytokeratin 19 and CD8, alongside a battery of other dry eye tests, in 27 GvHD patients with dry eye. Of these, 18 showed clinical signs of chronic oGvHD. In contrast to earlier research studies [44,45], this study failed to demonstrate a correlation between oGvHD status and HLA-DR expression in conjunctival epithelial cells [12]. The authors did, however, report an association between CD8-stained lymphocytes and oGvHD. Despite a small patient cohort, the results indicated the potential of CD8 as a biomarker for severe DED, particularly in oGvHD.

\section{Sjögren's syndrome-dry eye}

Sjögren's syndrome (SS) is the second most common autoimmune disease and in its primary form, SS is characterized by immune-mediated lymphocytic infiltration of the lacrimal and salivary glands, resulting in tissue destruction and fibrosis. Primary SS symptoms primarily present as severe dry eyes and dry mouth (xerostomia). 
Some groups have undertaken studies of patients with SS-dry eye (SS-DE), using IC, to ascertain if any associations exist between this sampling technique and clinical findings of DED. For example, a recent study by Mocanu et al. [46] assessed 42 subjects with SS-DE (vs 10 normals), to determine if cellular anomalies could be correlated with clinical parameters, with a view to specifically linking IC to SS-DE severity. All subjects underwent histopathological examination of ocular surface cells by IC of the superior conjunctiva, using cellulose acetate filter paper (Millipore), followed by fixation of cells in $95 \% / 1 \%$ ethanol:formalin, and periodic acid-Schiff (PAS) staining. Those found with SS-DE were divided into four groups of DED severity (stages I-IV; of increasing severity). This IC study revealed that normal subjects demonstrated numerous small, round epithelial cells with a nucleus/cytoplasm ratio of 1:2 and large PAS-positive goblet cell cytoplasms. In SS-DE patients, a variety of conjunctival epithelial morphologies were noted, depending on severity. For example, for low-grade severity, there were slightly enlarged epithelial cells, and smaller nuclei (nucleus/cytoplasm 1:3). With increasing sicca severity, epithelial cells were smaller and showed increasing signs of squamous metaplasia, as well as clumping of mucous. By contrast, the goblet cells in normal subjects were abundant, oval and demonstrated intense cytoplasmic staining for PAS. In SS-DE individuals, there was goblet cell loss and progressive epithelial cell flattening, which was significantly different to normals $(\mathrm{p}<0.001)$. The authors surmised that IC permitted rapid identification of conjunctival changes in SS-DE, with a sensitivity of $>90 \%$ and a specificity of $>95 \%$ in these patients, and showed a robust correlation between clinical severity and squamous metaplasia grade [46].

Similarly, Jirsova et al. [22] used IC on individuals with severe DED to determine levels of squamous metaplasia, except that in this research, the effects of autologous serum on cell changes were also investigated. The patient cohort was composed of six individuals with primary SS, six with secondary SS, one with sarcoidosis and four patients had DED of indeterminate cause. All subjects remained on their existing DED therapy, in addition to the autologous serum, throughout the study. The researchers measured established hallmarks of squamous metaplasia (goblet and epithelial cell densities) and noted evident cellular changes in IC, with severity of DED. For instance, significant increases in lower conjunctival goblet cell densities $(\mathrm{p}<0.0001)$ and epithelial cells densities $(\mathrm{p}<0.05)$, were observed, while significant reductions in HLA-DR positive cells $(\mathrm{p}=0.026)$, snake-like chromatin $(\mathrm{p}=0.022)$ and apoptosis $(\mathrm{p}=0.024)$ were observed after 3 months of autologous serum therapy. This study illustrated the usefulness of IC in measuring levels of squamous metaplasia in severe dry eye (including SS-DE), as well as in monitoring response to therapeutic regimes [22].

As well as using IC to monitor squamous metaplasia in severe DED, novel SS-DE biomarkers have been identified by gene analysis following RNA extraction from IC membranes. Recently, McNamara et al. [47] performed qPCR for paired box protein 6 (PAXG, an embryonic transcription factor involved in ocular mucosal surface development), $I L-1 \beta$ and SPRRIB (a pathological keratinization marker) on RNA from IC membranes of 43 SS-DE patients and 43 healthy controls. SS-DE patients revealed significantly reduced expression of PAXG $(\mathrm{p}=0.01)$, and PAX6 loss was strongly correlated with the ocular staining score $(\mathrm{p}=0.027)$. Moreover, this group confirmed that SPRR1B expression was a marker for pathological keratinization (commonly seen in SS), by reporting significantly increased expression in SS patients versus controls $(\mathrm{p}=0.009)$. Of note, the authors reported that SPRR1B increases correlated with higher ocular surface $I L-1 \beta$ levels $(\mathrm{p}<0.0001)$. Together, these results present convincing evidence of a link between PAX6 loss, local inflammation and squamous metaplasia in severe DED/SS-DE. Once again, gene analysis by IC demonstrates its usefulness in confirming previous animal study data in a human setting.

\section{Ocular allergies}

Conjunctival inflammatory conditions commonly include allergy-associated conjunctival disease, and can be grouped into conditions such as seasonal allergic conjunctivitis (SAC), vernal keratoconjunctivitis (VKC) and atopic keratoconjunctivitis (AKC). Patients with ocular surface allergies have shown evidence of increased eosinophils, which appear to mediate allergic conditions through release of histamine (via mast cell degranulation), as indicated by the presence of eotaxin, a potent chemoattractant for eosinophils, in their tear fluids [48].

A unique study using IC of conjunctival and corneal cells from subjects with AKC and corneal ulcers $(\mathrm{n}=8)$ was performed by Okada et al. [49] to analyze surface characteristics and the presence of inflammatory cell infiltrates. This group used cellulose acetate filter paper (Millipore) applied to the upper palpebral conjunctiva and corneal ulcer, followed by counterstaining with PAS. In samples containing advanced squamous metaplasia, they noted wide-spread invasion of eosinophils and neutrophils among ocular surface epithelial cells. As a result, the authors suggested that this IC-based clinical evidence of inflammatory cells served to demonstrate their hostile effects on 
corneal epithelial cells undergoing squamous metaplasia in AKC. They concluded that inflammatory infiltrates resulted in adverse effects on ocular surface cellular cohesion and integrity [49].

Another example of ocular allergy that has been investigated using IC is VKC, which is predominantly diagnosed by signs and symptoms, with the latter presenting as itching, tearing, redness and photophobia. Though it should be noted that these presentations are similar to other forms of allergic conjunctivitis and, within VKC, these symptoms may vary from patient-to-patient [50]. Therefore, objective tools may be necessary to confirm a VKC diagnosis. In recent years, pro-inflammatory mediators have been assessed by IC, in order to determine their involvement in mediating ocular surface allergies, such as VKC. For example, a study by Shoji et al. [51] investigated conjunctival mRNA expression of eotaxin-1, -2 and -3 in IC membranes of VKC patients (alongside tear protein analysis by ELISA). VKC subjects $(\mathrm{n}=25)$ were compared with normal healthy controls $(\mathrm{n}=11)$ using reverse transcriptase PCR and the authors reported a significantly higher positive ratio of eotaxin-1, -2 and -3 mRNA expression ( $\mathrm{p}<0.01, \mathrm{p}<0.01$ and $\mathrm{p}<0.05$, respectively) in the VKC group. Increased expression of this eotaxin subgroup in VKC indicated their potential as pathophysiological biomarkers of inflammatory cell infiltrates in this specific demographic of ocular surface allergy. While later work on VKC by Pong et al. [52] analyzed IC-derived human conjunctival cells (alongside tear proteomic assays) and demonstrated high levels of hemopexin expression (a class I acute-phase protein which is secreted after inflammatory events) via immunocytochemistry. Specifically in VKC patients, they reported increased cytoplasmic hemopexin in dekeratinized conjunctival epithelium and necrotic macrophages, but not in other inflammatory cells (eosinophils and neutrophils), thus indicating a role for this protein in the pathology of VKC [52]. Additionally, work by Inada et al. [53] utilized IC (nitrocellulose membranes) to analyze conjunctival epithelial cell mRNA of patients with VKC $(\mathrm{n}=11)$, versus normal healthy controls $(\mathrm{n}=11)$, alongside in vitro assays. qPCR of extracted RNA was used to assess expression of $I L-8$, eotaxin-2 and $C C L-20$ (a chemokine ligand for CCR6). The authors reported that CCL-20 mRNA expression was present in all conjunctival locations of controls. By contrast, significantly increased expression of $C C L-20$ was observed in the severe VKC group, versus the mild VKC and control groups $(\mathrm{p}<0.05)$. The authors surmised that CCL-2O, which is associated with inflammatory cell migration and the presence of inflammation, may mediate ocular surface homeostasis, hence worsening of VKC presentation [52]. Similarly, a recent IC study by Shiraki et al. [54] enrolled 18 patients with VKC or AKC (and 12 healthy controls) to assess eotaxin-2 mRNA levels by qPCR. The results showed significantly higher levels of eotaxin-2 in the active stage than for the stable stage of the AKC/VKC group and the controls (both $\mathrm{p}<0.01$ ), once again indicating eotaxin-2 as a putative biomarker of the active inflammatory stage of AKC/VKC.

\section{Dry eye disease \& other ocular conditions} Keratoconus

Keratoconus is a common degenerative and bilateral disorder that is characterized by thinning of the cornea, resulting in corneal distortion. It presents with bulging of the cornea, giving the distinctive conical shaping observed. Although commonly described as noninflammatory, some studies have reported impaired tear secretion and DED in keratoconus patients [55,56]. Subsequently, a pilot study by Carracedo et al. [57] investigated keratoconus patients $(\mathrm{n}=15)$ for the presence of DED, using morphological assessment of goblet cells by IC. They found that, when compared with healthy controls $(n=16)$, keratoconus patients demonstrated significantly lower densities of goblet cells $(\mathrm{p}<0.05)$, once more showing how IC may be reliably used to assist in confirming DED diagnoses. More recently, keratoconus research has employed conjunctival IC (alongside tear osmolarity, TBUT, Schirmer test, OSDI and ocular staining) to monitor ocular surface (and tear) changes, pre- and postcorneal collagen crosslinking [58]. This study of 26 patients with progressive keratoconus showed statistically significant increases in IC grading (superior and temporal; $p=0.005$ and $p=0.006$, respectively) at 3 months post-treatment versus pretreatment. However, it should be noted that both studies [57,58] are preliminary and comprise small patient numbers, therefore further studies are necessary to confirm these findings.

\section{Thyroid orbitopathy}

Thyroid orbitopathy (TO), also referred to Graves' orbitopathy or thyroid eye disease, is an autoimmune condition usually associated with thyroid disease, especially Graves' disease (GD). It presents with enlarged, dysfunctional extraocular muscles, strabismus and diplopia. Moreover, a common complication of TO is DED, which occurs due to increased proptosis and lower lid retraction (and thus exposed ocular surfaces), resulting in incomplete blinking and partial distribution of tears across the ocular surface. This causes enhanced evaporation of tears, hyperosmolarity 
and resultant ocular surface inflammation [59]. As a consequence of TO, it is imperative that markers of DED be identified in order to better manage this debilitating condition. A review of the literature concerning TO, ocular surface inflammation/DED and IC yielded a number of studies, some of which will be discussed here.

A study by Ismailova et al. [60] assessed 92 eyes from 46 patients with TO in order to ascertain morphological changes to the ocular surface. Following ocular surface damage (DED) being identified in 56 eyes (via positive lissamine green staining), these eyes underwent conjunctival histological analysis. Using IC, the authors reported goblet cell loss, extreme desquamation of superficial cells and epithelial keratinization. This group concluded that, although the conjunctival histopathological changes were consistent with DED, they were not specific for TO [60]. Similarly, Wei et al. [61] performed histopathological analysis (in vivo confocal microscopy) of IC membranes from 15 TO subjects versus 15 controls, to investigate changes to the conjunctiva. As seen by Ismailova et al. [60], Wei et al. [61] noted reduced bulbar conjunctival goblet cell density (superior and temporal, $\mathrm{p}<0.004$ and $\mathrm{p}<0.01$, respectively), as well as significantly increased squamous metaplasia in the TO group (both superior and temporal, $\mathrm{p}<0.001)$. Moreover, the latter study found significantly lower levels of superficial epithelial cell density in the superior bulbar conjunctiva, versus controls $(\mathrm{p}=0.002)$. Both studies appear to endorse the fact that IC can be used to confirm a clinical diagnosis of DED in patients with TO $[60,61]$. The question remains as to whether these findings are specific to TO and so this may be where the identification of other biomarkers of ocular surface inflammation, that are specific to the condition, comes into play.

As an example, Pawlowski et al. [62] undertook an IC and flow cytometric-based study of the previously described conjunctival epithelial cell inflammatory markers, HLA-DR and ICAM-1, in 10 subjects with active TO, versus 15 subjects with GD (without active TO) and 10 healthy controls. PES membranes were applied to the superior and superior-temporal bulbar conjunctiva and underwent cell extraction for flow cytometric analysis. Of interest, this group demonstrated a significant increase in HLA-DR positive conjunctival epithelial cells with active TO, versus GD without active TO and healthy controls (both $\mathrm{p}<0.005$ ). Furthermore, conjunctival epithelial cells from active TO patients showed significant increases in ICAM-1 versus controls $(\mathrm{p}<0.005)$. While patients with GD without active TO showed no increase in ICAM-1 expression, versus controls. These data indicate putative roles for both HLA-DR and ICAM as specific biomarkers of TO-associated ocular inflammation [62].

Recent work by Hsu et al. [15] utilized immunostaining of IC membranes to evaluate dry eye as a result of TO. Using immunofluorescence, membranes were stained for a number of DED-related pro-inflammatory cytokines, including IL-1 $\alpha$, IL-1 $\beta$ and IL-6. All subjects with TO-associated DED showed positive staining for these cytokines. Of interest was the finding that a trend occurred between the clinical inflammatory score and immunological IC grade, indicating these cytokines as potential biomarkers and/or therapeutic targets of TO-related dry eye. Although further research is needed to validate the findings of the latter two studies [15,62], the initial data are promising.

\section{Impression cytology \& systemic conditions associated with dry eye disease}

It should be noted that in recent years, IC has been used increasingly in the diagnosis of DED as a complication of systemic disease, such as cystic fibrosis $(\mathrm{CF})$, psoriasis and colitis.

\section{Cystic fibrosis}

Although CF is a hereditary disease, subjects may be affected by DED presentations as this disorder influences all secretory epithelia, including those in the eye. Early work by Mrugacz et al. [63] used IC and flow cytometry to assess the aforementioned inflammatory marker HLA-DR in conjunctival epithelial cells from patients with CF $(n=25)$ versus healthy controls $(n=25)$. This unique study demonstrated, for the first time, a significantly increased HLA-DR expression on CF patient conjunctival epithelial cells, versus normals $(p=0.0019)$. Moreover, the authors showed significant reductions in tear production and tear quality in CF patients $(\mathrm{p}<0.001$ and $\mathrm{p}<0.0001$, respectively) which, in combination, indicates an unstable tear fluid and resultant ocular surface inflammation. A subsequent study by this group [64] used IC to assess ocular surface health of CF patients. The authors reported a significant increase in squamous metaplasia grade in this specific patient demographic, versus controls $(\mathrm{p}=0.00013)$. Additionally, CF subjects revealed significantly lower average goblet cell densities than in the control groups ( $\mathrm{p}<0.001$ ), while inflammatory cells (neutrophils) were noted in four CF patients [64]. The additional information, which provided evidence of neutrophil infiltrates, also served to confirm the alreadyestablished inflammatory nature of dry eye, regardless of the underpinning primary disorder. The authors concluded that IC helped provide useful clues to the pathological changes in ocular surface cells of CF patients, which could be used to observe progression (and/or response to therapeutics) in DED of this specific patient cohort. 
Psoriasis

Psoriasis is a chronic autoimmune disease that primarily affects the skin, resulting in patches of a red, scaly rash, found on the scalp, ears, elbows and knees. In addition, psoriasis may present with ocular symptoms, specifically of the anterior segment [65]. Manifestations may include dry eye, conjunctivitis and blepharitis. To date, only two studies have employed IC to assess conjunctival cell morphological changes in psoriasis-associated DED [66,67]. However both studies have yielded important information. In 1999, Karabulut et al. [66] assessed 50 individuals with chronic plaque psoriasis and compared them to 50 healthy volunteers and reported that $50 \%$ of the psoriasis subjects had a grade $0,30 \%$ had a grade I and $20 \%$ had a grade II conjunctival IC differentiation, versus $95 \%, 3 \%$ and $2 \%$, respectively for controls $(\mathrm{p}<0.001)$. In addition, a snake-like appearance of nuclear chromatin cells was noted more commonly in eyes in the psoriasis group. Together, the use of IC served to identify early morphological changes to the conjunctiva in this patient group. A more recent study in 2013 [67] assessed 30 patients with chronic plaque psoriasis and 30 healthy controls via IC. The authors reported significant conjunctival cellular changes, as well as diminished goblet cell density $(\mathrm{p}<0.001)$ compared with controls $(\mathrm{p}=0.003)$. Thus, both studies showed significant morphological changes to the conjunctiva and ocular surface degeneration, leading to dry eye, in those with psoriasis.

\section{Colitis \& coeliac disease}

Finally, a recent study by Oltulu et al. [68] employed IC (nitrocellulose acetate) to assess DED associated with ulcerative colitis (UC). UC is a chronic condition which manifests as colonic mucosal inflammation and ulceration. This disorder also presents with numerous ocular complications, including dry eye, blepharitis, episcleritis and anterior uveitis [69]. In this novel study, samples from patients with UC $(n=15)$ were compared with healthy controls $(\mathrm{n}=15)$. IC of the conjunctiva revealed a statistically significant difference $(\mathrm{p}<0.05)$ in distribution of IC grades between the two groups. This group also showed significantly lower tear production and tear quality (Schirmer-I test and TBUT test) in patients with UC compared with the controls. This is the first study of its kind to show compromises to the tear fluid and conjunctival cells in subjects with inflammatory bowel disease, further confirming the usefulness of IC in diagnosis and monitoring of systemic disease-associated dry eye [69].

Similarly, research by Uzel et al. [70] used IC to assess ocular surface changes in coeliac disease (CD). CD is an immune-mediated inflammatory condition of the small intestine that is triggered by dietary intake of gluten. In this study, IC was performed on 56 eyes of 28 patients with CD versus 58 eyes of 29 healthy controls. A significant difference in IC grades between the two groups $(p=0.001)$ was observed. Moreover, patients with CD disease presented with larger conjunctival epithelial cells and fewer goblet cells. Thus CD appears to be related to morphological changes of the ocular surface which may present as dry eye. Both studies are unique in presenting such data and indicate a new area of research involving the use of IC which may shed light onto the ocular dysfunction associated with inflammatory bowel disease [70,71].

\section{Conclusion}

From the literature reviewed, it can be seen that IC has the potential to provide detailed cytological data in confirming a diagnosis of squamous metaplasia/dry eye, both in OSDs and in systemic diseases with an ocular involvement. In addition, vast improvements in the sensitivities of available gene and protein assays have allowed identification of a number of putative biomarkers that require further investigation.

Thus, the advantages of IC are:

- Much less invasive than conjunctival biopsies, scraping or brush cytology.

- Specific (or multiple) conjunctival areas can be sampled.

- Morphological data can be elucidated alongside biomarkers analysis.

- No disruption to the basement membrane.

- Provides a biochemical 'snapshot' of the tissue at that point in the disease course.

It should also be noted that IC also has a number of limitations, including:

- A lack of standardization in the methodology used (i.e., membrane type used, fixation procedures, areas sampled and processing/extraction techniques).

- Sampling is usually restricted to the outermost tissue layers.

- The effects of topical anesthetics on subsequent assays (e.g., qPCR) have not been fully examined. 
- Direct RNA/protein extraction of whole membranes may not permit identification of the exact cellular source of biomarkers (i.e., goblet or epithelial cells, or inflammatory cell infiltrates).

In summary, for systemic disease-related DED, it will be interesting to see if future IC studies identify specific biomarkers for each of the conditions reviewed here (e.g., cytokines and other immune mediators), to permit improved diagnosis, stratification and therapeutic surveillance, or if there are similarities to DED biomarkers of nonsystemic origin. Research within our group has indicated substantial inhibition of qPCR components, following IC retrieval after topical anesthetics [AGANET AL. UNPUBLISHED ]. Therefore future studies, including the optimization of standard operating procedures for qPCR, should first be undertaken in order to address this potentially confounding factor. As with tear fluid biomarker analysis [72], it is anticipated that future IC studies comprising larger patient cohorts, more strict stratification of recruited patients, improved assay sensitivities and standardized methodologies will all serve to enhance the diagnosis, monitoring and treatment of disorders of the ocular surface.

\section{Future perspective}

It can be seen that in the last decade, the utilization of IC in assessing ocular surface changes in DED have dramatically increased. Not only have studies presented evidence of histopathological changes to the conjunctiva, but improved sensitivities of protein-based and gene-based analysis have allowed the discovery of several putative biomarkers. Previous reviews have indicated the use of ocular surface tissues, including tears, for direct assessment of ocular diseases in directing personalized medicine [70,71]. It is therefore anticipated that further identification of other, more specific, biomarkers will lead to an improved understanding of the mechanisms underlying DED, as well as providing novel therapeutic targets for this complex and multifactorial disease.

Executive summary

\section{Impression cytology \& ocular surface disease}

- With ageing global populations, early diagnosis of ocular surface disease is necessary to avoid reduced quality of life and productivity.

- In the field of personalized medicine, impression cytology (IC) has the potential to confirm clinical diagnoses of ocular surface disease.

Dry eye disease $\&$ other ocular conditions

- Several groups of biomarkers for dry eye disease have been identified by IC, including cytokines, mucins and inflammatory markers which may also provide therapeutic targets.

- In keratoconus, studies have shown HLA-DR, ICAM-1 and cytokines as putative biomarkers.

Impression cytology \& systemic conditions associated with dry eye disease

- In subjects with systemic diseases cystic fibrosis, colitis and coeliac disease, changes to the conjunctival epithelium have been reported.

- However, as this is a new area of research, more specific cellular biomarkers have yet to be identified.

Conclusion

- IC represents a rapid, user-friendly technique for retrieving ocular surface cells for gene and protein analysis.

- Future, more standardized, studies are necessary to delineate biomarkers specific to systemic disease with ocular involvement.

Financial \& competing interests disclosure

S Hagan is employed by GCU, Scotland, UK, is a lecturer and PI in Vision Sciences in the Department of Life Sciences at GCU, with a special interest in predictive and prognostic biomarkers of ocular surface disease. The author has no other relevant affiliations or financial involvement with any organization or entity with a financial interest in or financial conflict with the subject matter or materials discussed in the manuscript apart from those disclosed.

No writing assistance was utilized in the production of this manuscript.

\section{Open access}

This work is licensed under the Attribution-NonCommercial-NoDerivatives 4.0 Unported License. To view a copy of this license, visit http://creativecommons.org/licenses/by-nc-nd/4.0/ 


\section{References}

Papers of special note have been highlighted as: $\bullet$ of interest; $\bullet \bullet$ of considerable interest

1. Thatcher RW, Darougar S, Jones BR. Conjunctival impression cytology. Arch. Ophthalmol. 95, 678-681 (1977).

2. Egbert PR, Lauber S, Maurice DM. A simple conjunctival biopsy. Am. J. Ophthalmol. 84, 798-801 (1977).

-. One of the primary and original publications in the area of impression cytology (IC).

3. Nelson DJ, Havener VR, Cameron JD. Cellulose acetate impressions of the ocular surface. Dry eye states. Arch. Ophthalmol. 101, 1869-1872 (1983).

4. Doughty MJ. Objective assessment of conjunctival squamous metaplasia by measures of cell and nucleus dimensions. Diagn Cytopathol. 39(6), 409-423 (2011).

- A study on the utility of IC in accurately measuring cell:nucleus ratios in the diagnosis of squamous metaplasia.

5. Danjo $\mathrm{Y}$, Watanabe $\mathrm{H}$, Tisdale AS et al. Alteration of mucin in human conjunctival epithelia in dry eye. Invest. Ophthalmol. Vis. Sci. 39(13), 2602-2609 (1998).

6. Pastor S, Rodriguez-Prats JL, Walewska-Szafran A, Rodriguez AE, Alio JL. Conjunctival impression cytology for the diagnosis, follow-up and treatment in a patient with severe keratoconjunctivitis caused by alkali injury. Cornea 28(7), 817-820 (2009).

7. López-Miguel A, Gutiérrez-Gutiérrez S, García-Vázquez C, Enríquez-de-Salamanca A. RNA collection from human conjunctival epithelial cells obtained with a new device for impression cytology. Cornea 36(1), 59-63 (2017).

- A novel study of RNA extraction using a new method of IC, the Eyeprim membrane.

8. Roy P, Cimbolini N, Antonelli $S$ et al. Assessment of a new device for conjunctival impression. Presented at: The ARVO Annual Meeting. Fort Lauderdale, FL, USA, 6-10 May 2012.

9. Blades KJ, Doughty MJ. Comparison of grading schemes to quantitative assessments of nucleus-to-cytoplasmic ratios for human bulbar conjunctival cells collected by impression cytology. Curr. Eye Res. 20(4), 335-340 (2000).

- A study on the utility of IC in accurately measuring cell:nucleus ratios in the diagnosis of squamous metaplasia.

10. Pisella PJ, Brignole F, Debbasch C et al. Flow cytometric analysis of conjunctival epithelium in ocular rosacea and keratoconjunctivitis sicca. Ophthalmology 107(10), 1841-1849 (2000).

11. Baudouin C, Haouat N, Brignole F, Bayle J, Gastaud P. Immunopathological findings in conjunctival cells using immunofluorescence staining of impression cytology specimens. Br. J. Ophthalmol. 76(9), 545-549 (1992).

12. Eberwein P, Issleib S, Böhringer D et al. Conjunctival HLA-DR and CD8 expression detected by impression cytology in ocular graft versus host disease. Mol. Vis. 19, 1492-1501 (2013).

13. Bradley JL, Edwards CS, Fullard RJ. Adaptation of impression cytology to enable conjunctival surface cell transcriptome analysis. Curr. Eye Res. 39(1), 31-41 (2014).

14. López-García JS, García-Lozano I, Rivas L, Giménez C, Acera A, Suárez-Cortés T. Effects of autologous serum eye drops in conjunctival expression of MUC5AC in patients with ocular surface disorders. Cornea 35(3), 336-341 (2016).

15. Hsu SL, Lee PY, Chang CH, Chen CH. Immunological impression cytology of the conjunctival epithelium in patients with thyroid orbitopathy-related dry eye. Genet. Mol. Res. 15(3), doi:10.4238/gmr.15039011 (2016).

16. Brignole-Baudouin F, Riancho L, Ismail D, Deniaud M, Amrane M, Baudouin C. Correlation between the inflammatory marker HLA-DR and signs and symptoms in moderate to severe dry eye disease. Invest. Ophthalmol. Vis. Sci. 58(4), 2438-2448 (2017).

17. Massingale ML, Li X, Vallabhajosyula M, Chen D, Wei Y, Asbell PA. Analysis of inflammatory cytokines in the tears of dry eye patients. Cornea 28(9), 1023-1027 (2009).

-• One of the earliest papers to use both IC and multiplex analysis of tear fluids to assess cytokine expression of patients with dry eye disease.

18. Hagan S, Omatayo B, Oliver K, Doughty M, Walshe C. An inflammatory gene profile of human conjunctival epithelial cells in dry eye disease. Presented at: The 8th International Conference on the Tear Film \& Ocular Surface: Basic Science and Clinical Relevance. Montpellier, France, 6-10 September 2016.

19. Wittpenn JR, Tseng SC, Sommer A. Detection of early xerophthalmia by impression cytology. Arch. Ophthalmol. 104(2), 237-239 (1986).

20. Liu X, Yu FF, Zhong YM, Guo XX, Mao Z. Therapeutic effects of sodium hyaluronate on ocular surface damage induced by benzalkonium chloride preserved antiglaucoma medications. Chin. Med. J. (Engl.) 128(18), 2444-2449 (2015).

21. Jee D, Park SH, Kim MS, Kim EC. Antioxidant and inflammatory cytokine in tears of patients with dry eye syndrome treated with preservative-free versus preserved eye drops. Invest. Ophthalmol.Vis. Sci. 55(8), 5081-5089 (2014).

22. Jirsova K, Brejchova K, Krabcova I et al. The application of autologous serum eye drops in severe dry eye patients; subjective and objective parameters before and after treatment. Curr. Eye Res. 39(1), 21-30 (2014).

23. Doughty MJ. Assessment of consistency in assignment of severe (grade III) squamous metaplasia to human bulbar conjunctiva impression cytology cell samples. Ocul. Surf. 13(4), 284-297 (2015). 
24. Versura P, Profazio V, Schiavi C, Campos EC. Hyperosmolar stress upregulates HLA-DR expression in human conjunctival epithelium in dry eye patients and in vitro models. Invest. Ophthalmol. Vis. Sci. 52(8), 5488-5496 (2011).

25. Baudouin C, Brignole F, Becquet F, Pisella PJ, Goguel A. Flow cytometry in impression cytology specimens. A new method for evaluation of conjunctival inflammation. Invest. Ophthalmol. Vis. Sci. 38, 1458-1464 (1997).

26. Yafawi R, Ko M, Sace FP, John-Baptiste A. Limitations of an ocular surface inflammatory biomarker in impression cytology specimens. Cutan. Ocul. Toxicol. 32(1), 46-53 (2013).

27. Epstein SP, Gadaria-Rathod N, Wei Y, Maguire MG, Asbell PA. HLA-DR expression as a biomarker of inflammation for multicenter clinical trials of ocular surface disease. Exp. Eye Res. 111, 95-104 (2013).

28. Moore QL, De Paiva CS, Pflugfelder SC. Effects of dry eye therapies on environmentally induced ocular surface disease. Am. J. Ophthalmol. 160(1), 135-142 (2015).

29. Brignole F, Pisella PJ, De Saint Jean M, Goldschild M, Goguel A, Baudouin C. Flow cytometric analysis of inflammatory markers in KCS: 6 month treatment with topical cyclosporin A. Invest. Ophthalmol. Vis. Sci. 42(1), 90-95 (2001).

30. Brignole-Baudouin F, Baudouin C, Aragona P et al. A multicenter, double-masked, randomized, controlled trial assessing the effect of oral supplementation of omega- 3 and omega- 6 fatty acids on a conjunctival inflammatory marker in dry eye patients. Acta Ophthalmol. 89(7), e591-e597 (2011).

31. Tsubota T, Fujihara K, Saito T. Takeuchi conjunctival epithelium expression of HLA-DR in dry eye patients. Ophthalmologica 213(1), 16-19 (1999).

32. Aronni S, Cortes $\mathrm{M}$, Sacchetti $\mathrm{M}$ et al. Upregulation of ICAM-1 expression in the conjunctiva of patients with chronic graft-versus-host disease. Eur. J. Ophthalmol. 16(1), 17-23 (2006).

33. Corrales RM, Narayanan S, Fernández I et al. Ocular mucin gene expression levels as biomarkers for the diagnosis of dry eye syndrome. Invest. Ophthalmol. Vis. Sci. 52(11), 8363-8369 (2011).

34. Zhang J, Yan X, Li H. Analysis of the correlations of mucins, inflammatory markers and clinical tests in dry eye. Cornea 32(7), 928-932 (2013).

35. Duncan K, Jeng BH. Medical management of blepharitis. Curr. Opin. Ophthalmol. 26(4), 289-294 (2015).

36. Lindsley K, Matsumura S, Hatef E, Akpek EK. Interventions for chronic blepharitis. Cochrane Database Syst. Rev. 5, CD005556 (2012).

37. Karalezli A, Borazan M, Dursun R, Kiyici H, Kucukerdonmez C, Akova YA. Impression cytology and ocular surface characteristics in patients with seborrhoeic dermatitis. Acta Ophthalmol. 89(2), e137-e141 (2011).

38. Watson SL, Daniels JT, Geerling G, Dart JK. Clinical trials of therapeutic ocular surface medium for moderate to severe dry eye. Cornea 29(11), 1241-1246 (2010).

39. Zhang L, Su Z, Zhang Z, Lin J, Li DQ, Pflugfelder SC. Effects of azithromycin on gene expression profiles of proinflammatory and anti-inflammatory mediators in the eyelid margin and conjunctiva of patients with meibomian gland disease. JAMA Ophthalmol. 133(10), 1117-1123 (2015).

40. Munir SZ, Aylward J. A review of ocular graft-versus-host disease. Optom. Vis. Sci. 94(5), 545-555 (2017).

41. Na KS, Kim MS. Allogeneic serum eye drops for the treatment of dry eye patients with chronic graft-versus-host disease. J. Ocul. Pharmacol. Ther. 28(5), 479-483 (2012).

42. Vanathi M, Kashyap S, Khan R et al. Ocular surface evaluation in allogenic hematopoietic stem cell transplantation patients. Eur. J. Ophthalmol. 24(5), 655-666 (2014).

43. Cocho L, Fernández I, Calonge $\mathrm{M}$ et al. Gene expression-based predictive models of graft versus host disease-associated dry eye. Invest. Ophthalmol. Vis. Sci. 56(8), 4570-4581 (2015).

44. Wang Y, Ogawa Y, Dogru M et al. Baseline profiles of ocular surface and tear dynamics after allogeneic hematopoietic stem cell transplantation in patients with or without chronic GVHD-related dry eye. Bone Marrow Transplant. 45, 1077-1083 (2010).

45. Kurpińska M, Turno-Krecicka A, Zajac-Pytrus H, Dziegiel P, Misiuk-Hojło M, Gorczyńska E. The role of inflammation in the progression of ocular surface damage in children following allogeneic haematopoietic cell transplantation. Folia Biol. (Praha) 59(4), 139-145 (2013).

46. Mocanu CL, Jurja S, Deca AG et al. Impression conjunctival cytology in sicca syndrome - correlations between clinical and histological findings related to dry eye severity. Rom. J. Morphol. Embryol. 57(1), 197-203 (2016).

47. McNamara NA, Gallup M, Porco TC. Establishing PAX6 as a biomarker to detect early loss of ocular phenotype in human patients with Sjögren's syndrome. Invest. Ophthalmol. Vis. Sci. 55(11), 7079-7084 (2014).

48. Leonardi A, Jose PJ, Zhan H, Calder VL. Tear and mucus eotaxin-1 and eotaxin-2 in allergic keratoconjunctivitis. Ophthalmology 110(3), 487-492 (2003).

49. Okada N, Fukagawa K, Takano Y et al. The implications of the upregulation of ICAM-1/VCAM-1 expression of corneal fibroblasts on the pathogenesis of allergic keratopathy. Invest. Ophthalmol.Vis. Sci. 46, 4512-4518 (2005).

50. Pattnaik L, Acharya L. A comprehensive review on vernal keratoconjunctivitis with emphasis on proteomics. Life Sci. 128, 47-54 (2015). 
51. Shoji J, Inada N, Sawa M. Evaluation of eotaxin-1, -2 and -3 protein production and messenger RNA expression in patients with vernal keratoconjunctivitis. Jpn. J. Ophthalmol. 53(2), 92-99 (2009).

52. Pong JC, Chu CY, Li WY et al. Association of hemopexin in tear film and conjunctival macrophages with vernal keratoconjunctivitis. Arch. Ophthalmol. 129(4), 453-461 (2011).

53. Inada N, Ishimori A, Shoji J. CCL20/MIP-3 alpha mRNA expression in the conjunctival epithelium of normal individuals and patients with vernal keratoconjunctivitis. Graefes Arch. Clin. Exp. Ophthalmol. 252(12), 1977-1984 (2014).

54. Shiraki Y, Shoji J, Inada N. Clinical usefulness of monitoring expression levels of CCL24 (eotaxin-2) mRNA on the ocular surface in patients with vernal keratoconjunctivitis and atopic keratoconjunctivitis. J. Ophthalmol. 2016, 3573142 (2016).

55. Zemova E, Eppig T, Seitz B et al. Interaction between topographic/tomographic parameters and dry eye disease in keratoconus patients. Curr. Eye Res. 39, 1-8 (2014).

56. Dienes L, Kiss HJ, Perényi K et al. Corneal sensitivity and dry eye symptoms in patients with keratoconus. PLoS ONE 10(10), e0141621 (2015).

57. Carracedo G, Recchioni A, Alejandre-Alba N et al. Signs and symptoms of dry eye in keratoconus patients: a pilot study. Curr. Eye Res. 40(11), 1088-1094 (2015).

58. KalkanAkçay E, Kilicarslan A, Uysal BS et al. Tear function and ocular surface alterations after accelerated corneal collagen cross-linking in progressive keratoconus. Eye Contact Lens 43(5), 302-307 (2016).

59. Gürdal C, Saraç O, Genç I, Kırımlıŏglu H, Takmaz T, Can I. Ocular surface and dry eye in Graves' disease. Curr. Eye Res. 36(1), 8-13 (2011).

60. Ismailova DS, Fedorov AA, Grusha YO. Ocular surface changes in thyroid eye disease. Orbit 32(2), 87-90 (2013).

61. Wei YH, Chen WL, Hu FR, Liao SL. In vivo confocal microscopy of bulbar conjunctiva in patients with Graves' ophthalmopathy. J. Formos. Med. Assoc. 114(10), 965-972 (2015).

62. Pawlowski P, Mysliwiec J, Mrugacz M et al. Elevated percentage of HLA-DR ${ }^{+}$and ICAM-1 ${ }^{+}$conjunctival epithelial cells in active Graves' orbitopathy. Graefes. Arch. Clin. Exp. Ophthalmol. 252(4), 641-645 (2014).

63. Mrugacz M, Zak J, Bakunowicz-Lazarczyk A, Wysocka J, Minarowska A. Flow cytometric analysis of HLA-DR antigen in conjunctival epithelial cells of patients with cystic fibrosis. Eye (Lond.) 21(8), 1062-1066 (2007).

-• The first published study to utilize IC to investigate conjunctival inflammatory markers in the systemic disease, cystic fibrosis.

64. Mrugacz M, Kasacka I, Bakunowicz-Lazarczyk A, Kaczmarski M, Kulak W. Impression cytology of the conjunctival epithelial cells in patients with cystic fibrosis. Eye (Lond.) 22(9), 1137-1140 (2008).

65. Aragona E, Rania L, Postorino EI et al. Tear film and ocular surface assessment in psoriasis. Br. J. Ophthalmol. doi:10.1136/bjophthalmol-2017-310307 (2017) (Epub ahead of print).

66. Karabulut AA, Yalvac IS, Vahaboglu H, Nurozler AB, Duman S. Conjunctival impression cytology and tear-film changes in patients with psoriasis. Cornea 18(5), 544-548 (1999).

67. Her Y, Lim JW, Han SH. Dry eye and tear film functions in patients with psoriasis. Jpn J. Ophthalmol. 57(4), 341-346 (2013).

68. Oltulu P, Oltulu R, Asil M, Satirtav G, Mirza E. Conjunctival impression cytology and dry eye in patients with ulcerative colitis: a pilot study. Eye Contact Lens. doi:10.1097/ICL.0000000000000380 (2017) (Epub ahead of print).

-. Highly novel pilot study using IC to demonstrate significant morphological changes in ocular surface cells from patients with inflammatory bowel disease.

69. Cury DB, Moss AC. Ocular manifestations in a community-based cohort of patients with inflammatory bowel disease. Inflamm. Bowel Dis. 16(8), 1393-1396 (2010)

70. Uzel MM, Citirik M, Kekilli M, Cicek P. Local ocular surface parameters in patients with systemic celiac disease. Eye (Lond.) 31(7), 1093-1098 (2017).

71. Hagan S, Martin E, Enríquez-de-Salamanca A. Tear fluid biomarkers in ocular and systemic disease: potential use for predictive, preventive and personalised medicine. EPMA J. 7, 15 (2016).

- Recent systematic literature review of the diagnostic and therapeutic potential of ocular surface tissues (e.g., tears), for noninvasively identifying novel biomarkers of ocular and systemic pathologies.

72. Hagan S, Tomlinson A. Tear fluid biomarker profiling: a review of multiplex bead analysis. Ocul. Surf. 11(4), 219-235 (2013). 
(

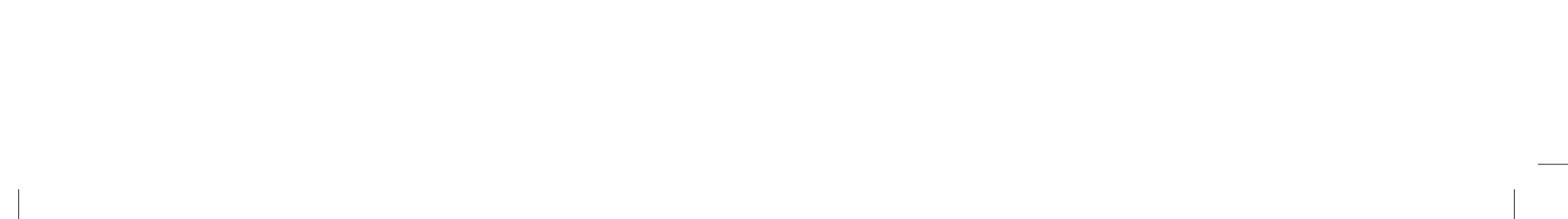

